\title{
The Challenge and promise of rare disease diagnosis in China
}

\author{
Xin $\mathrm{Ni}^{12^{* *}} \&$ Tieliu $\mathrm{Shi}^{3 *}$ \\ 'Otolaryngology, Head and Neck Surgery, Beijing Children's Hospital, Capital Medical University, National Center for Children's Health, \\ Beijing 100045, China; \\ ${ }_{2}^{2}$ Beijing Key Laboratory for Pediatric Diseases of Otolaryngology, Head and Neck Surgery, Beijing Pediatric Research Institute, Beijing \\ Children's Hospital, Capital Medical University, National Center for Children's Health, Beijing 100045, China; \\ ${ }^{3}$ The Center for Bioinformatics and Computational Biology, Shanghai Key Laboratory of Regulatory Biology, the Institute of Biomedical Sci- \\ ences and School of Life Sciences, East China Normal University, Shanghai 200241, China
}

Received May 16, 2017; published online June 14, 2017

Citation: Ni, X., and Shi, T. (2017). The Challenge and promise of rare diseases diagnosis in China. Sci China Life Sci 60, 681-685. doi: $10.1007 / \mathrm{s} 11427-017-9100-1$

Rare diseases are chronic and serious, featuring early onset at birth or in childhood, rapid deterioration and high mortality rate, which creates a burden on society and public health systems. Of the known rare diseases, 80 percent are genetic in origin, and half of those affected worldwide are children. In China, the rare disease patients are over 10 million, and 70 percent of the patients are children (Song et al., 2012; Liu et al., 2010).

Currently, the lack of knowledge, misdiagnoses, missed diagnoses and incurability have posed formidable obstacles to physicians and patients in effective diagnosis and treatment. In other words, getting to an accurate diagnosis can be a complicated, lengthy and frustrating journey for people with a rare disease as many physicians may only have limited experience with the identification and diagnosis of rare diseases. More importantly, the underlying biological mechanisms of a rare disease are often complex, making it difficult to undertake research and develop effective treatment strategies. In addition, due to the inherently small population of patients with a rare disease, recruiting for and conducting clinical studies can be difficult.

Tremendous efforts from academia, pharmaceutical companies, and government agencies have been made to accelerate and facilitate diagnosis and therapy for rare disease. For example, the USA Food and Drug Administration

*Corresponding authors (Xin Ni, email: nixin@ bch.com.cn; Tieliu Shi, email: tlshi@bio.ecnu.edu.cn)
(U.S. FDA) has added the Rare Pediatric Disease Priority Review Voucher Program (Section 529) to the Federal Food, Drug, and Cosmetic Act. Currently, more than 500 orphan drugs employed to treat about 250 kinds of rare diseases have been approved by the U.S. FDA since the Orphan Drug Act was passed in 1983, of which pediatric rare diseases accounted for a quarter. However, despite this progress, near $98 \%$ of rare diseases still do not have a single U.S. FDA-approved drug. Thus, there is a substantial unmet medical need for the treatments and diagnoses of rare diseases. In China, rare diseases have been paid muck less attention by health institutions, pharmaceutical companies and the society for a long time. At present, no rare disease treatment drugs have been developed domestically. The Chinese government has realized the shortage of resource in this field and begun to promote the basic researches and the collection of the rare disease information.

Generally speaking, rare diseases diagnosis and treatment is immature world-wide, especially there are still a lot of blanks on the study of pediatric rare diseases. Pediatric rare disease research is an important part of the 'Precision Medicine' which is becoming an emerging discipline. For many rare diseases, early diagnosis and treatment can obviously improve prognosis and life quality of patients.

To advance researches on the diagnosis and treatment of children's rare diseases, experts from Beijing Children's Hospital, Capital Medical University have joined with Sci- 
ence China Life Sciences journal to launch this thematic issue on about a dozen of those diseases and tried to dig into puzzles like the occurrence, development, diagnosis and treatment of the diseases, as an endeavor to explore possible breakthroughs.

In this Science China Life Sciences thematic issues, 10 articles contributed by different groups are dedicated to different pediatric rare diseases. Mitochondria are major centers for energy resource and many metabolic processes. Their gene dysfunctions can trigger various human diseases, many of which are rare ones. In this thematic issue, Drs. Ying Shen \& Fang Fang with their colleagues identified a mutation profile of many susceptible mitochondrial gens with a large cohort, many of those mutations are new ones and happened in the genes encoded by either mitochondrial DNA or nuclear DNA, which are linked to 8 different common or rare diseases (Fang et al., 2017).

As an X-linked recessive genetic disorder, androgen insensitivity syndrome (AIS) is a common cause of disorders of sex development and characterized by the variable degrees of female phenotypes of individuals with XY karyotype, and age-appropriate normal production of androgen by testes. AIS can be grouped into three categories-complete AIS (CAIS), milder degrees of under virilization (partial form or PAIS) or men with only infertility (mild form or MAIS), but no consensus criterion has been set for the clinical diagnosis of this disease by far. Dr. Gong led her group to test 28 patients of both CAIS and PAIS, they have detected new mutations in androgen receptor $(A R)$ gene, those mutation happened in ligand-binding domain (LBD), in the DNA binding domain (DBD) or a large N-terminal domain (NTD). The tested patients are either classified into CAIS or PAIS based on clinical manifestations, which indicated that other genes should also involve in the ASI and more systematic studies need be conducted to further explore the causative genes (Wang et al., 2017). Another work of Dr. Gong's group is to genome-wide analyze the DNA methylation pattern in Silver-Russell syndrome which is a clinically and genetically heterogeneous disorder with the incidence rate between 1/3,000 and 1/100,000. The high variability of its clinical manifestations hunger for the stable features for the disease diagnosis. By using various methods to detect the methylation patterns, they observed differentially methylated regions and confirmed that SRS patients show frequent DNA hypomethylation at imprinted gene OSBPL5 site cg25963939, suggesting that methylation changes are playing a role in the pathology of SRS and the new site could be potential feature for the disease diagnosis (Wu et al., 2017).

Pleuropulmonary blastoma (PPB) is a rare and highly aggressive mesenchymal neoplasm in childhood, although DICER1, which encodes a cytoplasmic endoribonuclease of the ribonuclease III (Rnase III) protein family to processe pre-miRNAs into mature miRNAs, has been discovered to be a causative genes of PPB, its pathogenesis is still not fully understood. Based on family history, Dr. Peng's group have detected various structure variations in DICERl genes from 12 consecutive cases of sporadic PPB, their results provided some new insight into the genetic and clinical characteristics of this disease (Cai et al., 2017).

The specialty of Drs. Ni and Guo is in pediatric Otolaryngology field, their group genotyped $B R A F$ gene among 48 pediatric patients with papillary thyroid cancer (PTC). They focused on BRAF V600E mutation and identified 17 patients carrying this mutation. Their findings indicate that BRAF V600E mutation is negatively correlated with partial aggressive clinicopathological features of the pediatric PTC. Considering that a large portion of the tested group do not carry the BRAF V600E mutation, other genes should also be involved in PTC and identification of those new genes with new mutations should benefit for the understanding of the underlying mechanism of the disease (Geng et al., 2017).

Rhabdomyosarcoma (RMS) is a rare, highly malignant soft tissue sarcoma in children with two major subtypes-Alveolar rhabdomyosarcoma (ARMS) and embryonal rhabdomyosarcoma (ERMS). Currently, the incidence of ARMS/ERMS is about 1.0/2.6 case per million children with age under 15 years. The choice of treatment for the disease is often directly determined by risk stratification, but sometimes ARMS and ERMS are difficult to be distinguished. To improve the clinical diagnosis of the disease, Drs. Ni and He with their team adopted fluorescence in situ hybridization (FISH) technique to detect the FOXO1 break-apart status and used it as biomarker to separate the ARMS and ERMS patients, and they found that signals of FOXO1 gene could distinguish atypical ARMS from atypical ERMS (Fu et al., 2017).

Blau syndrome is an autoimmune rare disease characterized by early onset of granulomatous inflammation of the skin, eye, and joints. Currently, only about 200 cases of Blau syndrome have been reported worldwide with no significant ethnic and gender predisposition. To better characterize this rare disease, $\mathrm{Dr}$. Li and her group analyzed mutation profile of CARD15 gene and the corresponding clinical features in 30 Chinese patients, they identified 10 missense mutations, 5 of them are novel ones. They summarized those detected mutations and clinical manifestations, which provide a new source for the right diagnosis of Blau syndrome (Li et al., 2017).

Bardet Biedl syndrome (BBS) is a rare multisystem disorder with clinical and genetic heterozygosity, over 21 candidate genes (BBS1-19, NPHP1, IFT172 et al.) have already been related to this disease. By using whole exome sequencing on a family with four members, Dr. Hui Wang's group identified compound heterozygous variants c.1192C>T, p.Q398* and c.1175C>T, p.T392M) in $M M K S$ gene which is likely responsible for the disease, they also proposed that the missense mutations in NPHPI and BBS9 gene might contribute to the mutation Load (Qiu et al., 2017). 
As an autosomal dominant genetic disorder involving multiple organs and systems, tuberous sclerosis complex (TSC) is a neurocutaneous syndrome with serious clinical presentations. To better characterize the association between genotypes and phenotypes of this disease, Dr. $\mathrm{Xu}$ Wang and her colleagues retrospectively investigated the clinical manifestations and genotypes of 20 Chinese children, and found that the mutation profiles in Chinese children are different from that in other countries. The mutations in TSC2 have more penetrative effect than that of TSC1, resulting in more severe clinical symptoms. Their results provide new information for the diagnosis and treatment of TSC (Bai et al., 2017).

Mutations in X-linked FRMD7 and GPR143 genes are likely associated with infantile nystagmus syndrome. By examining the FRMD7 gene mutation in 12 patients, Dr. Li $\mathrm{Li}$ and her group identified mutations and various structure variations in FRMD7 gene, most of which are deleterious. They also determined the relationship between waveform, visual acuity, and stereoscopic vision. Their summarized clinical information will facilitate the diagnosis of this disease in clinical application (Bai et al., 2017).

Overall, the lack of related clinical information, especially the detail description of the manifestation of the rare diseases make it much more difficult to promptly diagnose the rare diseases, we hope that the articles in this thematic issue can offer some helps to those related rare diseases in both diagnoses and treatments.

In the era of 'Precision Medicine', new technologies, especially the new generation sequencing, greatly facilitate our understanding of the underlying mechanism of rare diseases. However, it is still a long way to go to identify the causative genes for the rare diseases, since our data show that collectively, there are around 17,000 rare diseases, only about one third of them have clinical information available to certain extent and even less of them have been linked to corresponding pathogenic genes. Therefore, sharing the clinical information and related genetic variations of rare diseases becomes very important in the community, disseminating the clinical knowledge of rare diseases to public will significantly facilitate the accurate diagnosis of the rare diseases, ultimately, will promote the effective treatment of rare diseases.

To this end, we have teamed up to build a comprehensive integrative system-Encyclopedia of Rare Disease Annotations for Precision Medicine (eRAM) (www.pediascape. org/eram) with which we aim to help physicians and other clinical professionals in this community, as well as patients and their family members to gain knowledge of and more accurately diagnose rare diseases. In our eRAM system, we have standardized the rare disease names and clinical manifestations (phenotypes and symptoms) which make it much easier to exchange and integrate the different resources of clinical information.

Facing the challenge, National Institutes of Health (NIH) has established Undiagnosed Diseases Program (UDP) and tried to promote the rare disease diagnosis. However, it takes 8 to 12 weeks for UDP to evaluate an application, and the waiting list for admission is 2 to 6 months, there are only about $\sim 300$ cases accepted for the program each year. The progress of UDP is far more behind from the urgent needs of millions' families suffering from rare diseases. To alleviate the high demand of rare diseases diagnosis, we have built the related analysis pipeline, and also offer patients in poverty with the most puzzling medical significance free diagnosis. We encourage patients to submit their clinical information and contact us through eRAM system, we will help them identify those causative variations of diseases promptly, which in turn will benefit for the diagnosis of other patients with the same rare diseases.

Chinses government has made great efforts to improve people's health. Promoting children's health is crucial for building a healthy China, securing and improving people's livelihood, enhancing fitness for all, and realizing the Chinese Dream of national rejuvenation.

Beijing Children's Hospital, Capital Medical University is a time-honored and the largest top-level tertiary hospital providing comprehensive children's care that combines medical services, education, research, protection and prevention. During its 75-year history, Beijing Children's Hospital has been devoted to the public good, focusing on children's care and health, and making continuous and innovative efforts to serve as the guardian of children's health. In 2013, the hospital took the initiative in establishing Beijing Children's Hospital Group to explore national pediatric partnerships and give full play to its leading role. It then went further to build a non-profit social service organization engaging in pediatric development research-Futang Research Center of Pediatric Development, striving to contribute more to children's health through advancing diagnosis and treatment, supporting research and training talents. Beijing Children's Hospital topped the pediatric list in the 2016 ranking of Chinese hospitals' scientific and technological impact. When the year 2017 started, the National Health and Family Planning Commission issued an official document announcing the establishment of the National Center for Children's Health based in Beijing and with Beijing Children's Hospital, Capital Medical University as the mainstay, marking a milestone in children's health course in China.

Since 2012, the new management team has led the hospital in making rapid and profound progress in research. Supported by Capital Medical University, Beijing Children's Hospital and Beijing Pediatric Research Institute, the National Clinical and Research Center of Respiratory Diseases, Key Lab of Major Pediatric Disease Research under the Ministry of Education, National Scientific and Technology Cooperation Base for Major Pediatric Diseases, National Pediatric GCP, Futang Research Center of Pediatric Development and other national-level research platforms 
have been established to facilitate a research system equipped with state-of-the-art technologies and strong talent forces. In recent years, the hospital have been rewarded over 230 national, provincial and ministerial research projects, and hundreds of peer-review papers with academic influence published.

China has the largest population size in the world, which makes it possess a rich resource in various rare diseases. Beijing Children's Hospital, Capital Medical University has been committed to the diagnosis and research of children's rare diseases. Each year several million patients visit the hospital, and many children's rare diseases are not so rare to see in the hospital. To promote the researches, the hospital built the Biobank of Clinical Resources in Pediatrics in 2012. The biobank has collected hundreds of data items and supporting samples so far, providing rich resources for basic and clinical research of children's rare diseases and of great significance to understanding the pathogenesis and finding out potential clinical markers and drug targets.

Publishing this thematic issue is the first step to fulfill our commitment. Those updates in related rare diseases at molecular level is vitally significant, which not only are of benefit to the pediatric rare disease knowledge accumulation, but also can facilitate diagnosis and treatment for the corresponding rare diseases.

Stronger the Children, stronger a society. Children's health is where the Chinese dream starts.

Compliance and ethics The author(s) declare that they have no conflict of interest.

Bai, D.Y., Shi, W., Qi, Z., Li, W., Wei, A.H., Cui, Y.H, Li, C., and Li, L.
(2017). Clinical feature and waveform in infantile nystagmus syndrome in children with FRMD7 gene mutations. Sci China Life Sci 60, 707-713.

Bai, D.Y., Zhao, J.Y., Li, L., Gao, J, and Wang, X. (2017). Analysis of genotypes and phenotypes in Chinese children with tuberous sclerosis complex. Sci China Life Sci 60, 763-771.

Cai, S., Wang, X.S., Wen, Z., Fu, L.B., Ma, X.L., and Peng, X.X. (2017). DICER 1 mutations in twelve Chinese patients with pleuropulmonary blastoma. Sci China Life Sci 60, 714-720.

Fang, F, Liu, Z.M., Fang, H.Z., Wu, J., Shen, D.M., Sun, S.Z., Ding, C.H., Han, T.L., Wu, Y., Lv, J.L., Yang, L., Li, S.F., Lv, J.X., and Shen, Y. (2017). The clinical and genetic characteristics in children with mitochondrial disease in China. Sci China Life Sci 60, 746-757.

Fu, L.B., Jin, Y.Q, Jia, C., Zhang, J., Tai, J., Li, H.B., Chen, F., Shi, J., Guo, Y.L., Ni, X., and He, L.J. (2017). Detection of FOXO1 break-apart status by fluorescence in situ hybridization in atypical alveolar rhabdomyosarcoma. Sci China Life Sci 60, 721-728.

Geng, J.Q., Wang, H.M., Liu, Y.H., Jun Tai J., Jin, Y.Q., Zhang, J., He, L.J., Fu, L.B., Qin, H., Song, Y.L., Su, J.Z, Zhang, A.Y., Wen, X., Guo, Y.L., and Ni, X. (2017). Correlation of $B R A F^{\mathrm{V} 600 \mathrm{E}}$ and clinicopathological features in pediatric papillary thyroid carcinoma. Sci China Life Sci 60, 729-738.

Li, C.F., Zhang, J.M, Li, S.P., Han, T.X, Kuang, W.Y., Zhou, Y.F., Deng, J.H., and Tan, X.H. (2017). Gene mutations and clinical phenotypes in Chinese children with Blau syndrome. Sci China Life Sci 60, 758-762.

Liu, B.C., He, L., He, G., \& He, Y. (2010). A cross-national comparative study of orphan drug policies in the United States, the European Union, and Japan: towards a made-in-China orphan drug policy. J Public Health Policy 31, 407-420.

Qi, Z., Shen, Y., Fu, Q., Li, W., Yang, W., Xu, W.S., Chu, P., Zhang, Y.X., and Wang, H. (2017). Whole exome sequencing identified compound heterozygous variants in $M M K S$ gene in a Chinese pedigree with bardet biedl syndrome. Sci China Life Sci 60, 739-745.

Song, P., Gao, J., Inagaki, Y., Kokudo, N., and Tang, W. (2012). Rare diseases, orphan drugs, and their regulation in Asia: Current status and future perspectives. Intractable Rare Dis Res 1, 3-9.

Wang, Y., Gong, C.X., Wang, X., and Qin, M. (2017). AR mutations in 28 Patients with Androgen Insensitivity Syndrome Presented Prader 0-3. Sci China Life Sci 60, 700-706.

Wu, D., Gong, C.X., and Su, C. (2017). Genome-wide analysis of differential DNA methylation in Silver-Russell syndrome. Sci China Life Sci $60,692-699$.

\section{Biographical Sketch}

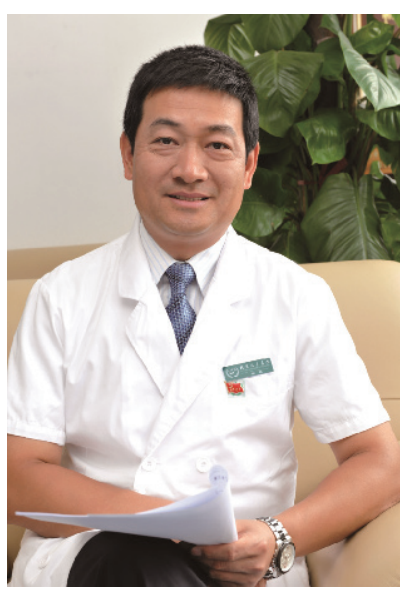

Dr. Xin Ni was graduated from Harbin Medical University and received his Bachelor of Medicine Degree in 1991. From 1991 to 2010, he worked in Beijing TongRen Hospital and successively had experience of surgical resident, attending surgeon, assistant to dean and deputy dean. During this period, he received Master of Medicine from Beijing TongRen Hospital \& Beijing Institute of Otolaryngology, and Master of Public Administration from Beijing University of Aeronautics and Astronautics in 2006. From 2010 to 2012, he worked in Beijing AnZhen Hospital as Deputy Dean. In 2012, Dr. Xin Ni received his Ph.D Degree from Harbin Medical University. Since 2012, Dr. Xin Ni was appointed as president of Beijing Children Hospital \& Beijing Pediatric Research Institute, professor of Department of Otolaryngology head and neck surgery, and chief of Beijing Biobank of Clinical Resources in Pediatric. Under President Ni's leadership, Beijing Children's Hospital Group was officially established on May 31, 2013, sharing pools of experts, clinical diagnosis, research, and training resources. It then went further to build Futang Research Center of Pediatric Development, with a total of 20 provincial level children's hospitals in 2016. The idea that "all Pediatrics departments nationwide belong to one system" is gradually being realized. In 2017, Beijing Children's Hospital has been approved as "National Center for Children's Health, China" by the National Health and Family Planning Commission. 


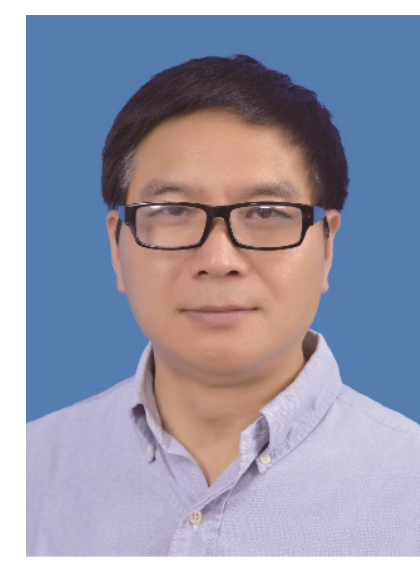

Dr. Tieliu Shi received his Master degree in Plant Physiology from Shanghai Institute of Plant Physiology, Chinese Academy of Sciences in 1992; Master degree in Computer Science in 1999 and Ph.D degree in Molecular Biology in 2000 from the University of Louisville, USA. After obtained Ph.D degree, he pursued bioinformatics research and joined the Bioinformatics Center, Shanghai Institute of Biological Sciences, Chinese Academy of Sciences between 2002 and 2008. He moved to East China Normal University by the end of 2008 and served as full professor. He has various research interests, including (i) clinical data standardization and integration analysis; disease gene, disease mechanism and biomarker discovery based on multi-level data integration of omics and clinical information; (ii) Methodology developments and applications in the high through-put data (NGS data, proteomic data, etc.); (iii) gene regulatory network prediction and protein-protein interaction network prediction; (iv) drug target, drug efficacy and adverse prediction, including Traditional Chinese Medicine. He has published more than 100 peer-review papers. 